\title{
The superiority of combined continuous wave Doppler examination over periorbital Doppler for the detection of extracranial carotid disease
}

\author{
U TROCKEL,${ }^{*}$ M HENNERICI, ${ }^{*}$ A AULICH,${ }^{*}$ W SANDMANN $\dagger$ \\ From the Departments of Neurology* and Surgery A, $\dagger$ University of Düsseldorf, West Germany
}

SUMMARY Non-invasive examination of 431 vessels in 333 patients with cerebrovascular disease in all stages was performed in order to compare the reliability of the periorbital Doppler test alone and together with the more difficult insonation of the carotid arteries in the neck (combined Doppler). These findings were compared with those of subsequent arteriography. Extracranial obstructive $(>50 \%)$ carotid disease was detected with $100 \%$ sensitivity by the combined Doppler, but with only $48 \%$ sensitivity by the periorbital indirect test. The specific ability of both methods to identify non-stenotic carotid arteries $(<50 \%)$ was similar at about $98 \%$. Only the combined Doppler examination reliably-differentiated various degrees of obstruction, comparable to that obtained with arteriography. Non-obstructive plaques could not be detected or excluded by either Doppler test. More refined methods will be necessary for their evaluation.

In the last decade various techniques have been reported for the non-invasive diagnosis of atherosclerotic lesions within the extracranial part of the carotid arteries. Some of these, like periorbital Doppler examination, are based on the well known retrograde perfusion of the ophthalmic artery via branches of the external carotid artery (supratrochlear and supraorbital arteries) in the presence of severe stenosis or occlusion of the extracranial internal carotid artery. However, although these indirect tests can easily be performed with rather simple, low cost technical equipment, their diagnostic reliability is restricted to the detection of haemodynamically severe obstructions (>80\% stenosis) within the proximal part of the internal carotid artery, in the absence of a significant stenosis of the external carotid artery. ${ }^{1-4}$ Therefore, supplementary direct recordings from the carotid arteries in the neck have been reported as necessary for a more accurate evaluation of less obstructive lesions, as well as for the exact estimation of their degree and site. ${ }^{5-9}$ This study attempted to compare

Address for reprint requests: Professor $M$ Hennerici, Neurologische Universitätsklinik, Moorenstr. 5, D-4000 Düsseldorf 1, West Germany.

Received 20 November 1982 and in revised form 14 May 1983 Accepted 5 July 1983 the clinical usefulness of the periorbital and the combined Doppler techniques, on the basis of 431 subsequently performed angiograms in 333 patients with cerebrovascular disease in all stages (asymptomatic, transient ischaemic attacks and strokes).

\section{Methods}

A directional continuous wave Doppler device with a nonfocused beam at $4 \mathrm{MHz}$ (Débimètre ultrasonique directionnel Delalande) was used for all recordings.

\section{Periorbital Doppler}

The flow curves of the supratrochlear and supraorbital arteries were recorded and sequential compression tests of the superficial temporal and facial arteries were carried out bilaterally. Compression of these branches of the external carotid artery should normally result either in no change or in augmentation of flow in the fronto-orbital branches of the ophthalmic artery. Reversal, alternating or zero flow in these arteries or a marked decrease of orthograde flow after compression of the external carotid artery branches indicate the presence of a severe obstruction of the proximal internal carotid artery (fig 1).

\section{Combined Doppler}

In addition to these indirect tests the flow curves of the carotid arteries were recorded throughout that part of their course in the neck which was accessible to direct insonation. The probe was initially positioned over the common carotid artery just above the clavicle; moving the probe in a cephalic direction along the course of the carotid system 

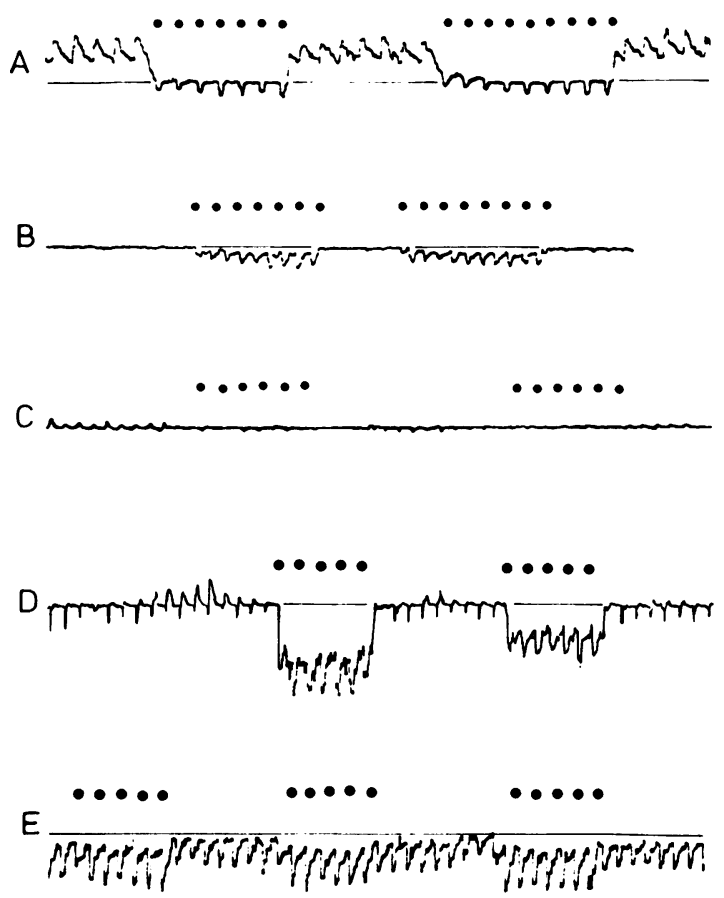

Fig 1 Doppler velocity profiles of the supratrochlear arteries showing considerable variations due to different changes of the haemodynamic conditions within the watershed between these branches of the internal carotid artery and external carotid artery. Recordings obtained from five patients with ipsilateral internal carotid occlusion at the bifurcation. (A) retrograde flow (upward deflexion) may change into orthograde flow (downward deflexion) after compression of facial branches of the external carotid artery (...). (B) zero flow with change into orthograde flow after compression manoeuvres. (C) small retrograde or orthograde flow without marked change following compression manoeuvres. (D) alternating flow with change into orthograde flow. (E) normal orthograde flow with slight increase after compression tests.

near the anterior border of the sternocleidomastoid muscle, the bifurcation as well as both the internal and external carotid arteries could be followed and clearly distinguished by differences in the audible characteristics of the blood flow signal and the distinct pulse curve characteristics recorded (fig 2). Additionally systematic compression manoeuvres on distal branches of the external carotid artery could be performed to identify the artery under consideration. ${ }^{10}$ This was essential in those cases where the existence of a continuous flow in branches of the external carotid artery may simulate an internal carotid flow (for example, occlusion of the internal carotid artery with functional external carotid artery collateralisation).

On the basis of our experience with more than 20000

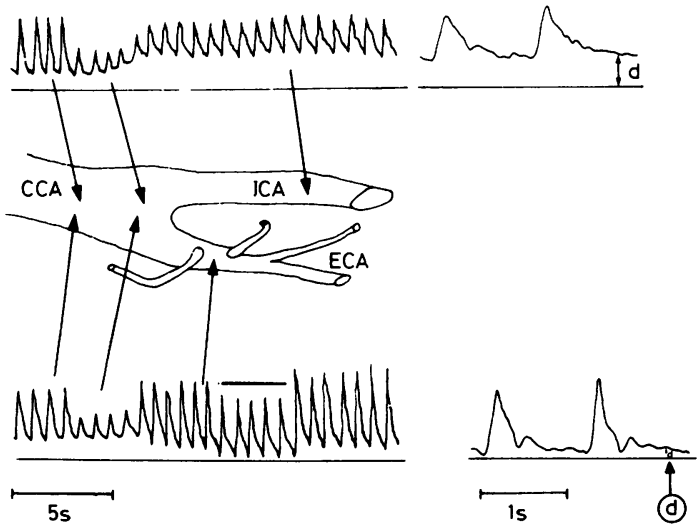

Fig 2 Normal Doppler velocity curves of the common (CCA), internal (ICA) and external carotid arteries (ECA) with characteristic differences of systolic amplitude modulation and continuous diastolic flow (" $d$ ") recorded by moving the probe along the extracranial course of the carotid system in the neck.

combined Doppler examinations in cerebrovascular patients during the last seven years, and comparison with subsequent angiographic and intraoperative results, ${ }^{68}$ five major Doppler findings can be differentiated in the carotid system:

1. Normal: Moving the probe from the common carotid artery to the bifurcation results in a reduction of peak systolic and diastolic flow velocity due to the widening of the vessel lumen. The velocity curves of the internal carotid artery and external carotid artery vary considerably (fig 2), according to the different circulatory resistances of the tissues they supply: the low resistance of the brain ensures that the diastolic pressure maintains a large continuous flow in the internal carotid artery and to some extent in the common carotid artery too, whereas the areas supplied by the external carotid artery have a much higher resistance, consequently reducing the diastolic component of flow ("d" in fig 2) almost to zero. These selective flow alterations can be demonstrated easily in the Doppler velocity curves recorded and can be unequivocally detected from the audible Doppler signals.

2. Extracranial Stenoses: Moving the probe from upstream to downstream across the carotid system in the neck may result in the detection of (a) a local increase in flow velocity (fig 3 ), demonstrated by an increase in the mean Doppler frequency ("mild stenosis"); (b) an increase in flow velocity associated with considerable distortion of the normal pulsatile flow velocity curves, and the appearance of a post-stenotic peak systolic flow deceleration (fig 4) corresponding to the low frequency vibration of the arterial walls and an area of flow instability distal to the stenosis with audible turbulence phenomena ("moderate stenosis"); (c) in addition to (b), the detection of an asymmetric flow in the common carotid arteries with reduction of the steady flow ipsilateral to the carotid obstruction, indicating the presence of a "severe stenosis" associated 

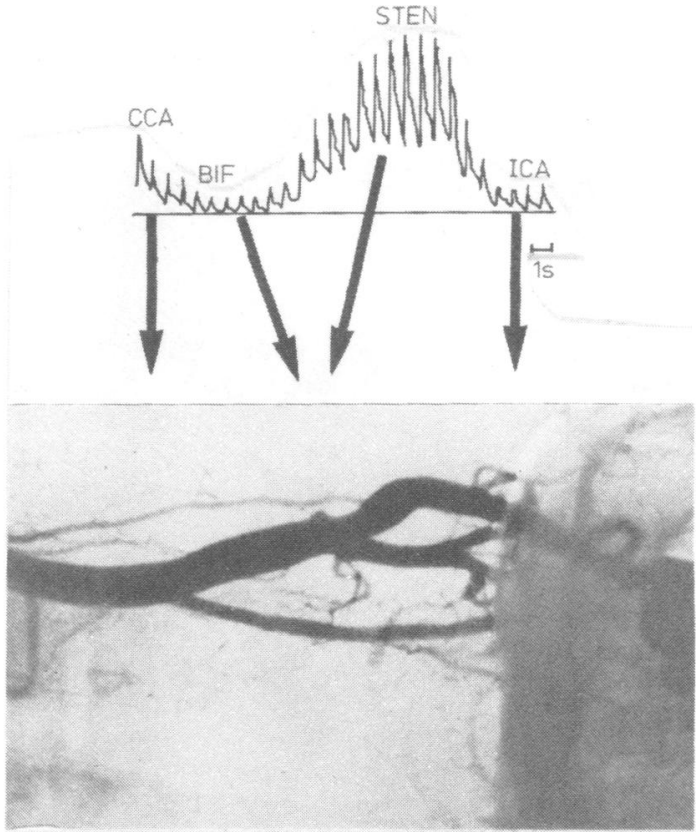

Fig 3 Typical Doppler velocity curves and corresponding arteriogram of a "mild" stenosis of the proximal internal carotid artery.

with an increase in circulatory resistance. Consequently the poststenotic flow is considerably reduced, and this frequently results in flow changes in the ophthalmic artery due to shifting of the watershed between the vascular areas of the internal and external carotid arteries (fig 1). These
Doppler flow curve alterations can be frequently demonstrated in the presence of internal carotid artery stenosis as well as of external carotid artery and common carotid artery stenoses which, however, occur less frequently.

3. Extracranial Occlusions: Occlusions of the internal carotid artery at the bifurcation are characterised by the findings that these vessels cannot be recorded, with reduction of flow in the proximal common carotid artery and frequent changes in the flow conditions of the ophthalmic artery (fig 1). Since in such circumstances flow conditions in the external carotid artery often resemble those normally seen in the internal carotid artery only, identification of each artery recorded in the neck by external carotid artery compression manoeuvres is diagnostic. Thrombosis of the internal carotid artery above the bifurcation may rarely show an alternating flow into the proximal stump of the vessel (forward flow during systole followed by a diastolic backflow).

4. Intracranial Obstructions: Obstructions of the intracranial arteries can only be detected by the combined Doppler examination if they induce an increase in the circulatory resistance. Thus only severe obstructions of the intracranial carotid and in the trunk of the middle cerebral arteries can sometimes be detected (fig 5) in the presence of steady flow asymmetry between the right and left extracranial common carotid arteries, internal carotid arteries and the branches of the ophthalmic arteries.

5. Nonstenotic Lesions: Early non-stenotic atheroma of the extracranial carotid system can only occasionally be detected by audible turbulence in the presence of marked alterations of the normal laminar blood flow conditions. With a zero-crossing Doppler flowmeter the signal curves recorded in such circumstances are indistinguishable from normal cases. Thus in this situation only, the diagnosis is crucially dependent on the interpretation of the audible signal by the examiner, unless more refined
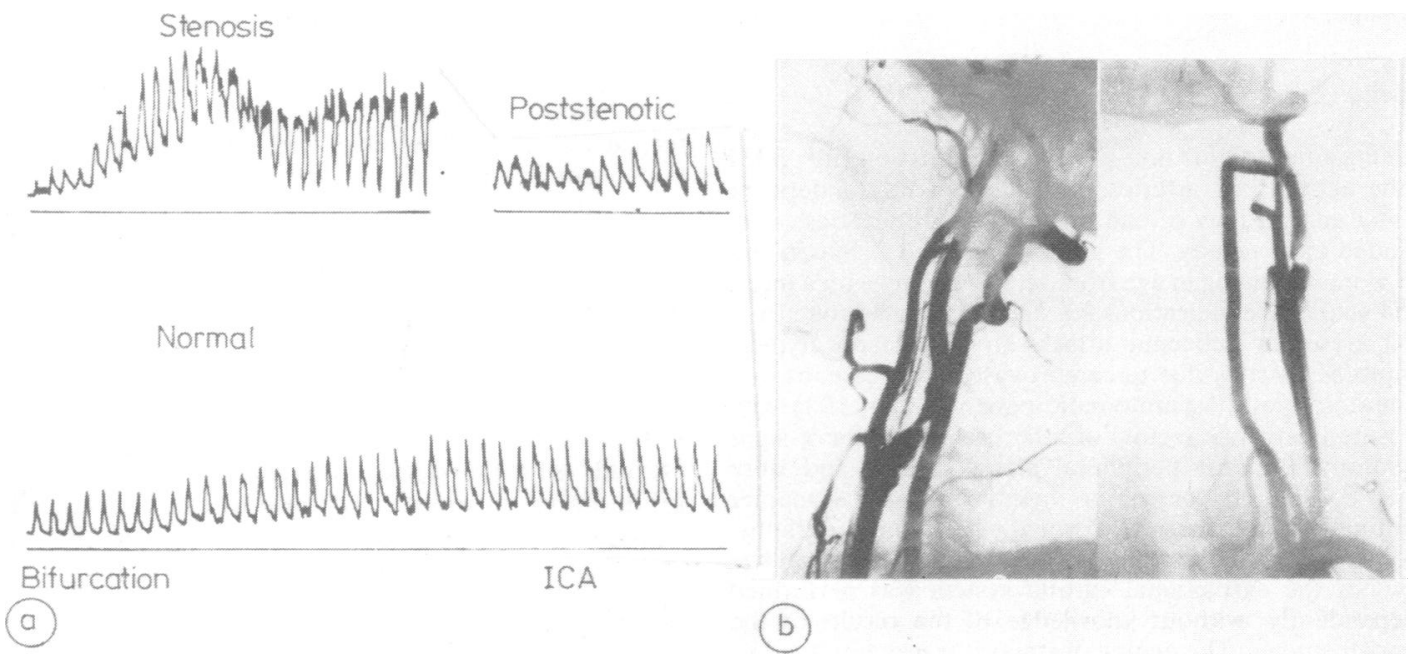

Fig 4 Typical Doppler velocity curves and corresponding arteriogram of a "moderate" stenosis of the proximal internal carotid artery. 


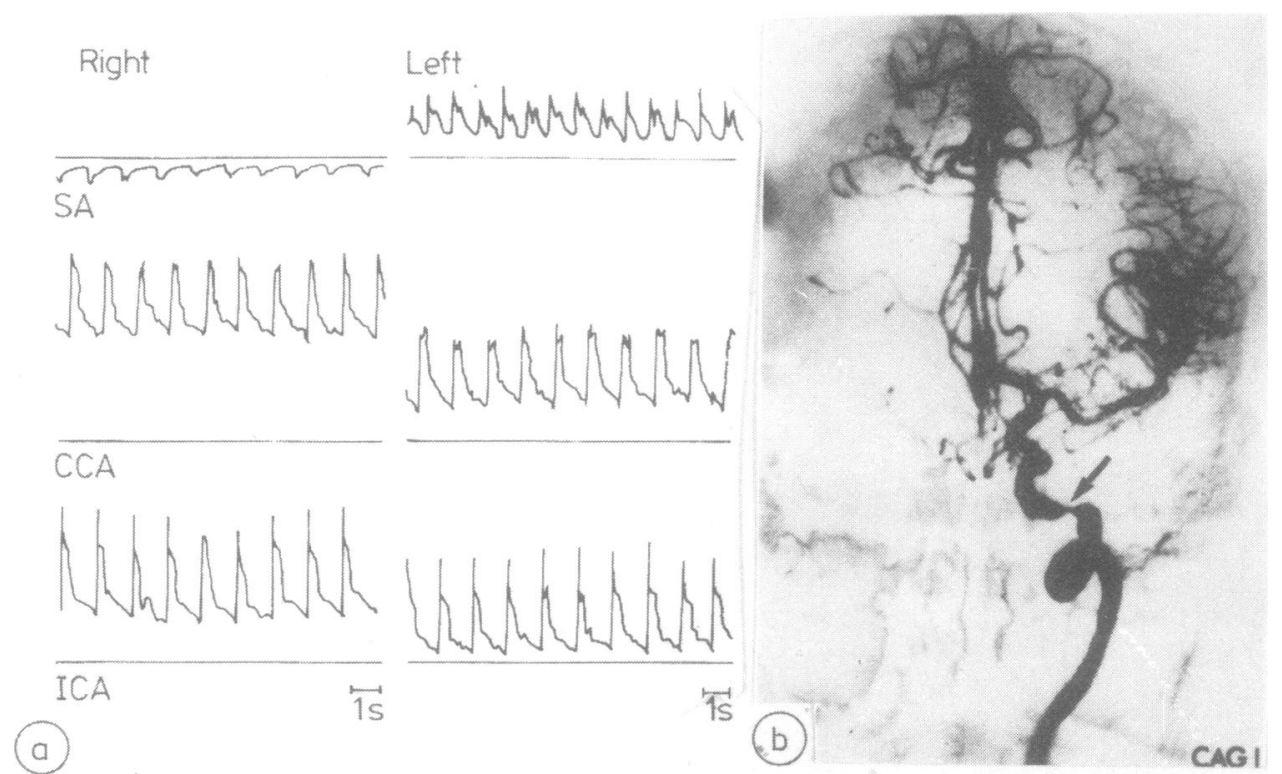

Fig 5 Typical Doppler velocity curves and corresponding arteriogram of a "severe" stenosis of the left distal internal carotid artery not accessible to direct examination but readily detectable in the presence of flow asymmetries of the common (CCA), internal carotid (ICA) and supratrochlear (SA) arteries.

examination techniques are introduced. ${ }^{11-14}$

Although the vertebral and subclavian arteries were always recorded in each patient, in order to obtain a systematic evaluation of the extracranial cerebral circulation, the results of these tests are excluded from the analysis in this paper.

\section{Patients}

Patients admitted for non-invasive examination $(n=333)$ of the extracranial arteries, who subsequently underwent carotid angiography of one or both carotid arteries, were included in this study. The group comprised 218 men and 115 women ranging in age from 18 to 81 years with a mean of 64 years. The indications for study included stroke $(n=$ $189)$, transient ischaemic attacks $(n=41)$ and atypical symptoms possibly due to cerebrovascular disease, as well as neurologically asymptomatic patients $(n=103)$ who presented with risk factors of atherosclerosis and/or signs of coronary and/or peripheral artery disease and were being considered for major vascular surgery. Doppler examination was always performed prior to arteriography. Interpretation of the contrast arteriograms with biplane views of the extracranial carotid system was performed independently, without knowledge of the results of the Doppler studies. The degree of stenosis was determined by measurements of the diameter of the most severe area of narrowing on a single plane film, with the degree of stenosis expressed as a percentage of the diameter reduc- tion relative to the normal distal vessel. This classification included six categories: normal, non-stenotic plaques $(<50 \%), 50-60 \%, 60-80 \%,>80 \%$ stenosis and blockage. Based on this classification the results of the Doppler methods were expressed in terms of sensitivity and specificity. Sensitivity was defined as that proportion of truly abnormal arteries (established by arteriography) that was considered to be abnormal by Doppler examination, expressed as a percentage. The sensitivity was determined for each category. Specificity was defined as that proportion of angiographically normal or only equivocally diseased carotid arteries that were normal at Doppler examination. Non-stenotic lesions were either separately listed (table 1) or excluded as "true negatives" (table 2), in order to avoid artificial inflation of the non-invasive test.

\section{Results}

Different degrees of obstruction within the extracranial internal carotid artery could be reliably graded and localised according to the characteristic changes in the combined Doppler velocity features already mentioned, as shown by corresponding arteriographic examination (table 1).

The overall sensitivity of combined Doppler examination in detecting different degrees of stenosis or occlusion $(>50 \%)$ was $100 \%$, its specificity $99 \%$ : all of the 210 obstructive lesions 
Table 1 Comparison between results of combined Doppler and carotid arteriography on 431 vessesls in 333 patients

\begin{tabular}{|c|c|c|c|c|c|c|c|}
\hline \multirow{3}{*}{$\begin{array}{l}\text { Extracranial } \\
\text { carotid arteriogram }\end{array}$} & \multirow{3}{*}{$\begin{array}{l}\text { No. of } \\
\text { vessels }\end{array}$} & \multicolumn{5}{|c|}{ Combined Doppler } & \multirow[b]{3}{*}{ Occlusion } \\
\hline & & \multirow[b]{2}{*}{ Normal } & \multirow[b]{2}{*}{ Non-stenotic } & \multicolumn{3}{|c|}{ Stenosis } & \\
\hline & & & & Mild & Moderate & Severe & \\
\hline $\begin{array}{l}\text { Normal } \\
<50 \% \text { stenosis } \\
50-60 \% \text { stenosis } \\
60-80 \% \text { stenosis } \\
>80 \% \text { stenosis } \\
\text { Occlusion }\end{array}$ & $\begin{array}{r}168 \\
53 \\
48 \\
43 \\
65 \\
54\end{array}$ & $\begin{array}{r}162 \\
34\end{array}$ & $\begin{array}{r}6 \\
18\end{array}$ & $\begin{array}{r}1 \\
39 \\
1\end{array}$ & $\begin{array}{r}7 \\
40 \\
3\end{array}$ & $\begin{array}{r}2 \\
2 \\
56 \\
2\end{array}$ & $\begin{array}{r}6 \\
52\end{array}$ \\
\hline
\end{tabular}

had been correctly detected prior to angiography and only one showing a non-stenotic plaque in the arteriogram was suspected of suffering from a mild stenosis in the Doppler test.

Although the sensitivity of combined Doppler in predicting the degree of stenosis subsequently proven by arteriography was only $81 \%$ for mild $(50-60 \%), 93 \%$ for moderate $(60-80 \%), 86 \%$ for severe $(>80 \%)$ stenosis and $96 \%$ for total occlusion, a detailed comparison shows the following: in six cases the degree of stenosis shown by direct Doppler examination seemed to be less pronounced than that seen at arteriography. In four patients this may have resulted from estimating the degree of obstruction according to the most severe narrowing seen on the arteriogram. In two other patients the more likely explanation was a progression of the disease during the interval of more than 6 weeks between the two examinations; in these cases a severe internal carotid artery stenosis had progressed to total block at the time the arteriogram was performed.

In 18 cases the haemodynamic evaluation showed a degree of stenosis more marked than that indicated by the arteriogram. The majority of these patients presented with rather heterogenous bilateral carotid lesions. While the degree of the more severe obstruction closely corresponded with the arteriogram, combined Doppler examination falsely suggested a more severe contralateral lesion as a result of an increase in collateral flow. This caused a marked deterioration of the carotid flow changes, which disappeared after removal of the more severe stenosis. Consequently non-invasive re-examination after unilateral carotid surgery showed a better correspondence between arteriography and combined Doppler findings in evaluating the degree of the persisting, less severe stenosis.

A differentiation between total and subtotal occlusion of the internal carotid artery was correct in most of the cases examined. In six cases the Doppler examination falsely indicated total occlusion of the internal carotid artery, since neither flow disturbances at the stenotic site nor a markedly diminished flow distal to the sub-total internal carotid artery obstruction could be reliably identified. In three of these cases the internal carotid artery stenosis may have been missed because of coincidental severe external carotid artery stenosis, whereas in the remaining three cases the internal carotid artery stenosis was so extreme that the artery seemed to be totally occluded even at carotid angiography. Only the application of more sophisticated angiographic techniques, recently described by Countee et al, ${ }^{15}$ revealed the correct diagnosis. However, important limitations of combined Doppler examination which should be taken into account are: (1) its misinterpretation of total block of the internal carotid artery in the presence of a subtotal stenosis and (2) its poor sensitivity (34\%)

Table 2 Comparison between the results of periorbital and combined Doppler vs. arteriography for the detection of extracranial carotid obstructive lesions (>50\%)

\begin{tabular}{|c|c|c|c|c|}
\hline \multirow{2}{*}{$\begin{array}{l}\text { Extracranial } \\
\text { carotid arteriogram }\end{array}$} & \multicolumn{2}{|c|}{ Periorbital Doppler } & \multicolumn{2}{|c|}{ Combined Doppler } \\
\hline & No stenosis & Stenosis & No stenosis & Stenosis \\
\hline $\begin{array}{l}\text { No stenosis } n=221 \\
\text { Stenosis } n=210 \\
\text { Sensitivity } \\
\text { Specificity } \\
\text { Test accuracy } \\
\text { Pos. pred. val. } \\
\text { Neg. pred val. }\end{array}$ & $\begin{array}{l}217 \\
109\end{array}$ & $\begin{array}{r}4 \\
101\end{array}$ & 220 & $\begin{array}{rl} & 1 \\
100 \% & 210 \\
99 \% \\
99 \% \\
99 \% \\
100 \%\end{array}$ \\
\hline
\end{tabular}




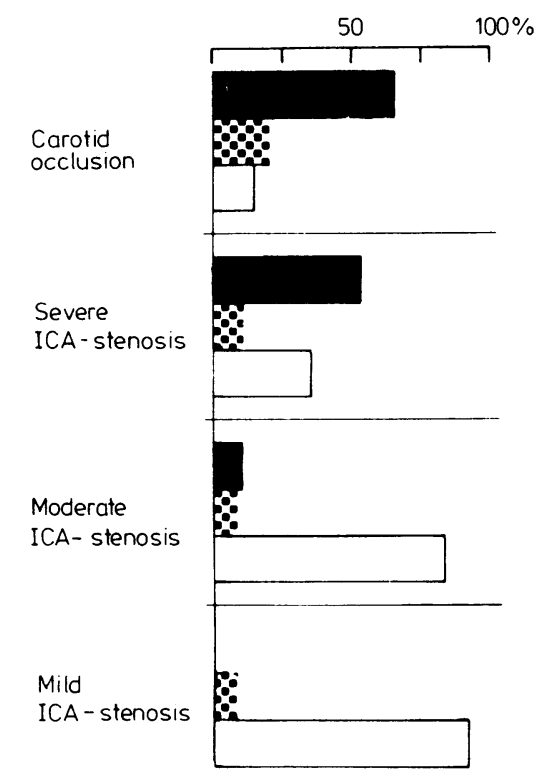

Fig 6 Summary of the incidence of various Doppler velocity features recorded from the supratrochlear arteries for various degrees of carotid obstruction: 1 retrograde flow. $\square$ zero-or alternating flow. $\square$ orthograde flow.

in the detection of non-stenotic plaques associated with its inability to distinguish ulcerative from nonulcerative lesions.

In comparison with the results of combined Doppler examination, periorbital Doppler proved to be a rather poor method for the reliable detection of extracranial internal carotid artery lesions (fig 6).

In cases with mild stenosis of the internal carotid artery a high percentage $(93 \%)$ showed a normal orthograde flow in the supratrochlear artery. In 7\% no flow was present. None showed retrograde flow. Among the group with moderate stenosis, retrograde or zeroflow was present in only $10 \%$ and $8 \%$ respectively. Among the group with severe stenosis and occlusion, retrograde flow was found in $53 \%$ and $64 \%$ respectively and zero flow was obtained in $10 \%$ and $21 \%$ respectively. Thus a correct diagnosis would have been missed in $93 \%$ of mild, $82 \%$ of moderate, $37 \%$ of severe arterial lesions and in at least $15 \%$ of occlusions, if the change in flow at the watershed of the anastomosis between the branches of the external carotid artery and those of the ophthalmic artery from extra- to intraorbital had been taken as the only diagnostic guide. Furthermore, none of the non-stenotic carotid lesions $(n=53)$ could be detected with this indirect technique, because it showed without exception normal ortho- grade flow. Since flow changes in the ophthalmic artery do not correspond with the actual degree of carotid obstruction (fig 1) but reflect the collateral capacity to develop different anastomotic pathways, the overall sensitivity of periorbital Doppler examinations for the detection of stenosis $(>50 \%)$ and occlusion on the basis of either zero, alternating, or retrograde flow was $48 \%$, the specificity of the test was $98 \%$ (table 2). If the comparison between periorbital Doppler examination and arteriography is restricted to severe stenosis $(>80 \%)$ and occlusion only, the sensitivity of the test $(63 \%$ and $85 \%$ respectively) still remains considerably inferior to that demonstrated by the combined Doppler examination.

\section{Discussion}

The increasing awareness of cerebrovascular disease caused by extracranial arterial lesions and the improved techniques for their surgical treatment have highlighted the need to identify those patients who might benefit from carotid endarterectomy. Selection of these patients is based on a correct interpretation of the history, neurological signs and symptoms and an adequate knowledge of the underlying vascular process. The latter frequently remains undetected until either cerebral embolism or haemodynamic insufficiency produces transient ischaemic attacks or stroke, usually indicating the need for arteriography. ${ }^{16}$ Thus there is a lack of reasonable criteria for the accurate management of those patients who present with atypical histories and ambiguous neurological signs. ${ }^{16}$ Moreover, the more CT examinations are performed, the greater is the number of patients with "silent cerebral infarction" requiring appropriate diagnosis and treatment.

Although arteriography provides a reliable assessment of extracranial arterial lesions, its hazards and impracticability for follow-up studies have led to the search for equally reliable but noninvasive techniques, in particular to compensate for the present large number of misleading results from carotid bruit auscultation. ${ }^{1718}$ The application of these techniques seems to be particularly helpful not only in patients with an uncharacteristic clinical picture, but also in those who are quite well apart from severe risk factors or the manifestation of generalised atherosclerosis (peripheral and coronary artery disease), and who reveal more frequently than hitherto expected quite severe extracranial asymptomatic arterial disease. ${ }^{816}$ Among the non-invasive techniques recommended during the last decade periorbital Doppler examination has come into widespread use particularly because it is easy to per- 
form. However, the usefulness of this indirect test is shown to be restricted to the detection of severe obstructive $(>80 \%)$ lesions which cause a marked reduction of blood flow within the distal carotid system and the ophthalmic artery (for review see ref 18). Since circulation within the ophthalmic artery may still be adequately supplied from the contralateral internal carotid artery or both vertebral arteries via the circle of Willis, this test may fail to yield a correct diagnosis in the presence of severe stenosis or occlusion if normal flow of the ipsilateral supratrochlear and supraorbital arteries is sufficiently maintained. The failure to detect a mild or moderate internal carotid artery stenosis results, for the same reasons, in an overall sensitivity of only $48 \%$. Thus periorbital Doppler examination is more likely to indicate the collateral capacity of the external carotid artery than to demonstrate the presence of a proximal internal carotid artery obstruction.

The results presented in this study show that direct examination of the neck arteries considerably improves the diagnostic value of periorbital Doppler examination as indicated by a similar specificity (99\% and $98 \%$ respectively), but a sensitivity amounting to $100 \%$ for the detection of extracranial obstructive carotid disease, even if mild and moderate lesions are included. Moreover, the degree of stenosis under consideration can be reliably graded and its site precisely localised.

A correct diagnosis of non-stenotic atherosclerotic plaques cannot be made with either technique, although a skilful examiner may be able to detect some of these minor irregularities of the arterial wall from audible turbulences on direct Doppler assessment of the neck vessels. Further refinements of the non-invasive test, such as computerised analysis of the Doppler frequencies, imaging procedures for the detection of minor tissue abnormalities and combinations of both in more complex systems, have already been developed and quite successfully used.11-14 However, at present these systems are very expensive, their clinical application is more difficult and time-consuming, and, most important, their advantages in the assessment of early atherosclerotic lesions are counterbalanced by a number of disadvantages in the detection of obstructive lesions. ${ }^{14} 19$ Thus in our experience the application of combined Doppler examination today represents the best method for the initial and follow-up examination of patients with definite and questionable cerebrovascular disease presenting with unusual signs and symptoms, even if the more refined methods are available. Although it may take some time to become acquainted with the technique and interpretation of combined Doppler examination, this skill should be as thoroughly acquired as other techni- ques (for example EMG analysis). With this proviso, invasive arteriography can be dispensed with in quite a number of patients. ${ }^{16} 20$ Its application, if necessary, may be more individually planned at the right time with a view to better treatment and follow-up.

We are grateful to Dr PRD Humphrey, London for helpful comments on the manuscript. We thank Mrs C Schlund and Mrs C Edler for technical assistance and Mrs B Kosemetzky for secretarial assistance. This work was supported by grants from the Deutsche Forschungsgemeinschaft SFB 200/D 2.

\section{References}

${ }^{1}$ Müller HR. Diagnosis of internal carotid artery occlusion by directional Doppler sonography of the ophthalmic artery. Neurology (Minneap) 1971; 22: 816-23.

${ }^{2}$ Büdingen HJ, Hennerici M, Voigt K, Kendel K, Freund H-J. Die Diagnostik von Stenosen oder Verschlüssen der A. carotis interna mit der direktionellen Ultraschall-Doppler-Sonographie der A. supratrochlearis. Disch Med Wischr 1976;101:269-75.

${ }^{3}$ Bone GE, Barnes RW. Clinical implications of the Doppler cerebrovascular examination: a correlation with angiography. Stroke 1976;7:271-4.

${ }^{4}$ Ackermann RH. Perspective on non-invasive diagnosis of carotid disease. Neurology (Minneap) 1979;29: 615-22.

5 Planiol TH, Pourcelot L, Pottiers JM. Etude de la circulation carotidienne par les methodes ultrasoniques et la thermographie. Rev Neurol (Paris) 1972;126: 127-42.

${ }^{6}$ v. Reutern G-M, Büdingen HJ, Hennerici, M, Freund $\mathrm{H}$-J. Diagnose und Differenzierung von Stenosen und Verschlüssen der A. carotis mit der DopplerSonographie. Arch Psychiat Nervenkr 1976;222: 191-207.

' Spencer MP, Reid JM. Quantitation of carotid stenosis with continuous-wave (cw) Doppler ultrasound. Stroke 1979;10:326-30.

${ }^{8}$ Hennerici M, Aulich A, Sandmann W, Freund H-J. Incidence of asymptomatic extracranial arterial disease. Stroke 1981;12:750-8.

9 Barnes RW, Nix L, Rittgers SE. Audible interpretation of carotid Doppler signals. Arch Surg 1981;116: 1185-9.

${ }^{10}$ Büdingen $\mathrm{HJ}, \mathbf{v}$ Reutern G-M, Freund H-J. Die Differenzierung der Halsgefäße mit der DopplerSonographie. Arch Psychiat Nervenkr 1976;222: 177-90.

"Blackshear WM, Phillips DJ, Chikos PM, Harley JD, Thiele BL, Strandness DE. Carotid artery velocity patterns in normal and stenotic vessels. Stroke 1980;11:67-71.

12 Woodcock JP. Doppler ultrasound in clinical diagnosis. Br Med Bull 1980;36:243-8. 
${ }^{13}$ Felix RW, Siegel B, Gibson RJ, Williams J, Popky GL, Edelstein AL, Justin JR. Pulsed Doppler ultrasound detection of flow disturbances in arteriosclerosis. $J$ Clin Ultrasound 1976;4:275-82.

${ }^{14}$ Hennerici M. Nicht-invasive Diagnostik des Frühstadiums arteriosklerotischer Carotisprozesse mit dem Duplex-System. VASA 1983;12:228-32.

${ }^{15}$ Countee RW, Vijayanathan, T. Reconstruction of "totally" occluded internal carotid arteries. $J$ Neurosurg 1979;50:747-57.

${ }^{16}$ Hennerici M, Rautenberg W, Mohr S. Stroke risk from symptomless extracranial arterial disease. Lancet 1982;ii:1180-3.

${ }^{17}$ Ziegler DK, Zileli T, Dyck A, Sebaugh JL. Correlation of bruits over the carotid arteries with angiographi- cally demonstrated lesions. Neurology (Minneap) 1971;21:860-5.

${ }^{18}$ Ginsberg MD, Cebul RD. Non-invasive diagnosis of carotid artery disease. In: Harrison JG, Dyken ML, eds. Cerebral Vascular Disease. London: Butterworths Pub, 1983:215-53.

${ }^{19}$ Diener HC. Continuous Wave Dopplersonographie und Duplex-Scan: zwei sich ergänzende, atraumatische Verfahren in der Diagnostik extrakranieller Stenosen und Verschlüsse. Electromedica, 1983;51:6-13.

${ }^{20}$ Sandmann W, Hennerici M, Nüllen H, Aulich A, Knab K, Kremer K. Carotid artery surgery without angiography: risk or progress? In: Greenhalgh RM, Rose FC, eds, Progress in Stroke Research II. London: Pitman, 1983:447-60. 